DOI: https://doi.org/10.14311/TPFM.2021.015

\title{
BOUNDARY LAYER ON THE FLAT PLATE WITH ROUGHNESS STRIP
}

\author{
Vladislav Skála ${ }^{1}$ \\ ${ }^{1}$ Institute of Thermomechanics of the Czech Academy of Sciences, Dolejškova 1402/5, \\ 182 00, Praha 8, Czech Republic
}

\begin{abstract}
Effect of the roughness strip on the boundary layer development on a flat plate was studied experimentally. A special model of a flat plate was designed. Experiment was made in nonperturbed flow. Smooth flat plate (with super elliptical leading edge) was equipped with short roughness strip (close to the leading edge). Profiles of total pressure along the boundary layer were measured using flattened Pitot tube. First basic results of experiments are presented.
\end{abstract}

Keywords: boundary layer, transition to turbulence, roughness strip

\section{Introduction}

Grainy particles or surface disturbances such as coarse surface structure, tops or cavities (insect, corrosion products, flow erosion) on wings and blades are often present close to the leading edges when operated in real conditions. The determination or prediction of the start and size of the transitional zone of the boundary layer transition to turbulence is very important during design process of these parts.

The transition process can be characterised by the start point and end point of transitional zone, i.e. the region along a boundary layer, when it is changing from laminar (or "pseudolaminar") boundary layer into turbulent boundary layer. Better description of the boundary layer transition in specific conditions, location and size of the transition zone by transition from laminar boundary layer to turbulence, seems to be important for CFD simulations and transition models testing.

Two cases of boundary layers on a flat plate with influence of two different roughness strips were studied. Strips with roughness P32 and P100 (particles $/ \mathrm{cm}^{2}$ ) were placed on a smooth flat plate just behind the rounded "supereliptic shaped" leading edge. This paper presents the first results of this experimental investigation.

\section{Flat plate with roughness strip}

The flow over a smooth flat plate with roughness strip close to the leading edge can be used as a test or reference case for modelling of the influence of the surface roughness (particles deposition or local degradation of the surface) on the boundary layer, transitional process and skin friction respectively. Such a case is very common in energetics (wind turbines) or aerodynamics of aircraft (especially laminar airfoil of a wing).

Knowledge of quantitative and qualitative influence of this kind of disturbance close the leading edge is very important as it strongly influences beginning and size of the transitional zone. Roughness strip can prevent the boundary layer separation and it influences skin friction. Increased roughness close the leading edge turbulises the boundary layer. IT means, that the roughness strip can stabilise the flow. Stability of the flow is very important parameter for controllability of an airplane, especially at « lower » Reynolds numbers as in case of small airplanes (ULL and LSA airplanes, drones) during take-off and landing, at wrong control inputs or spin warning ability of airplanes. The disadvantage of using the roughness strip is increased skin friction coefficient leading to additional losses of useful energy under specific conditions, $[1-6]$.

\section{Experiments}

\subsection{Experimental setup}

The experiments were carried out in the closed type wind tunnel. The investigated boundary layers were developing on the model of flat plate, see Figure 1. Cross section of the test section of the wind tunnel was $0.9 \mathrm{~m} \times 0.5 \mathrm{~m}$, operated under standard conditions (air pressure and temperature). The length of the 
flat plate was $2.65 \mathrm{~m}$. The leading edge of the flat plate had a super-ellipse shape MSE6, length of the leading edge was $0.05 \mathrm{~m}$. Leading edge was followed with one roughness strip (magnetically fixed in a cavity). The length of the strips was $0.03 \mathrm{~m}$. Roughness was sand shaped ( $\mathrm{K}$ - type) of the size P100 and P32 (particles $/ \mathrm{cm}^{2}$ ). Flat smooth surface follows behind the roughness strip. Arrangement of the experiment is shown in Figure 1.

Experiment was realized at zero pressure gradient along the flat plate (except the small area close to the leading edge). The mean velocity was set to approx. $8 \mathrm{~m} / \mathrm{s}$, reference dynamic pressure $p_{\mathrm{d} R E F}$ indicated by Pitot - static pressure probe was controlled and held at the value of $43 \mathrm{~Pa}$. Natural free stream turbulence was $I u_{\mathrm{e}}=0.005$.

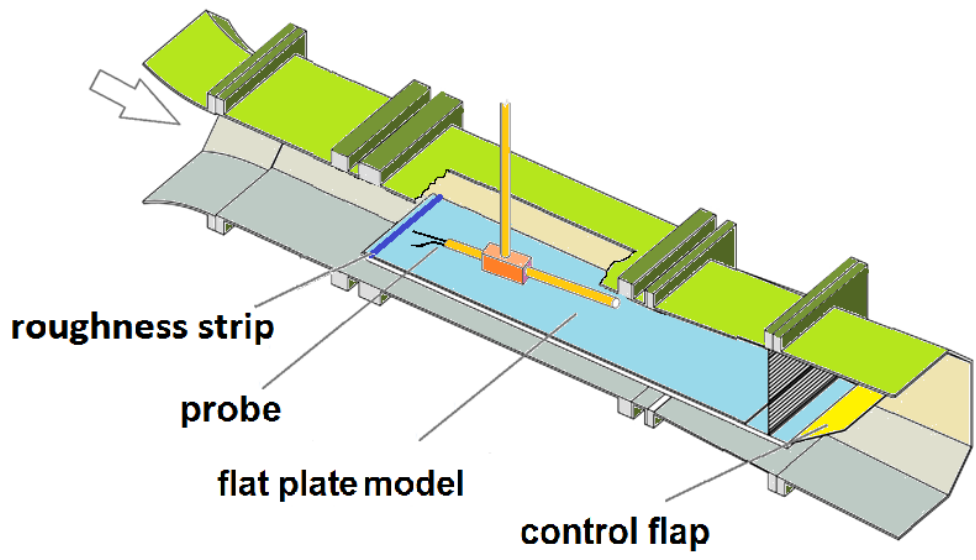

Figure 1: Experimental setup - flat plate in the wind tunnel test section.

\subsection{Measurement techniques}

Flow in the boundary layer was studied by special combined flattened Pitot (total pressure) - static pressure probe, see Figure 2. The size of the head of the flattened total pressure probe was $1.5 \cdot 10^{-5} \mathrm{~m}$. This probe is used to study the flow field with strong velocity gradients, such as boundary layer flow fields. The probe must be positioned perpendicular to the flow direction, the total pressure $p_{\mathrm{t}}$ and static pressure $p_{s}$ have to be on the same static pressure line. Then it is possible to evaluate local dynamic pressure $p_{\mathrm{d}}$ (as a difference of the total pressure $p_{\mathrm{t}}$ and static pressure $p_{\mathrm{s}}$ ) corresponding to the local velocity inside the boundary layer. The value of dynamic pressure was corrected when the probe Reynolds number $R e_{p}$ was bellow 100, see [7].

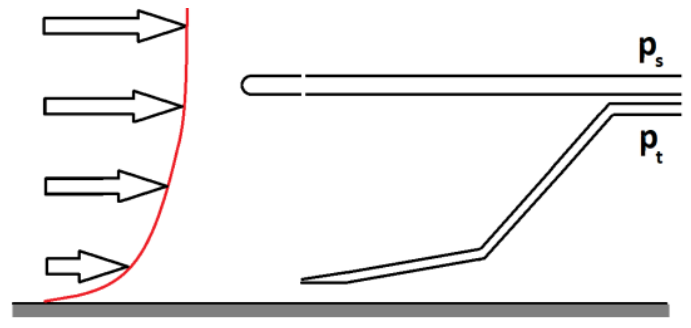

Figure 2: special flattened Pitot - static pressure probe.

The probe was put into defined positions by the computer controlled traversing device. The vertical distance $y$ (measured from the surface of plat plate) was checked manually by optical measurement using cathetometer with accuracy $\pm 0.02 \mathrm{~mm}$.

The reference value of the free stream velocity was measured by a Prandtl pressure probe (diameter $0.006 \mathrm{~m}$ ) at the inlet of the test section. Pressures hoses from probes and barometric pressure were connected to pressure transducers Druck DPI 145. The flow temperature was measured by thermometer Pt100. Output voltage was read by data acquisition unit HP 34970 A.

The output of all devices was recorded using National Instruments data acquisition system and recorded to the PC in LabView 2015 software. Time of one measurement point was set to $20 \mathrm{~s}$ according to the expected stability of the flow in the test section of the wind tunnel. 
Data was evaluated real-time using the LabView scripts and final results were checked and organised using MS Excel software.

\subsection{Measurement uncertainties}

The experimental uncertainties $\Delta$ are estimated based on relative errors of evaluated quantities. They depend mainly on accuracy of location of the probe (mainly the distance $y$ of the probe from surface of the flat plate) and accuracy of pressures measurements:

$$
\begin{gathered}
\Delta p_{d} / p_{\mathrm{d} R E F}= \pm 0.02 \\
\Delta y / y_{0}= \pm 0,01 \\
\Delta H_{12}= \pm 0.1
\end{gathered}
$$

\subsection{Basic boundary layer characteristics}

The boundary layer state can be described using the local mean velocity profiles measured across the boundary layer (perpendicular to the surface of the plat plate) in specified distances from the leading edge. The development of the boundary layer can be demonstrated as the comparison of the velocity profiles in dimensionless form, typically laminar, turbulent or transient shape of the mean velocity profile can be observed, some examples from experiment are plotted in Figure 3.

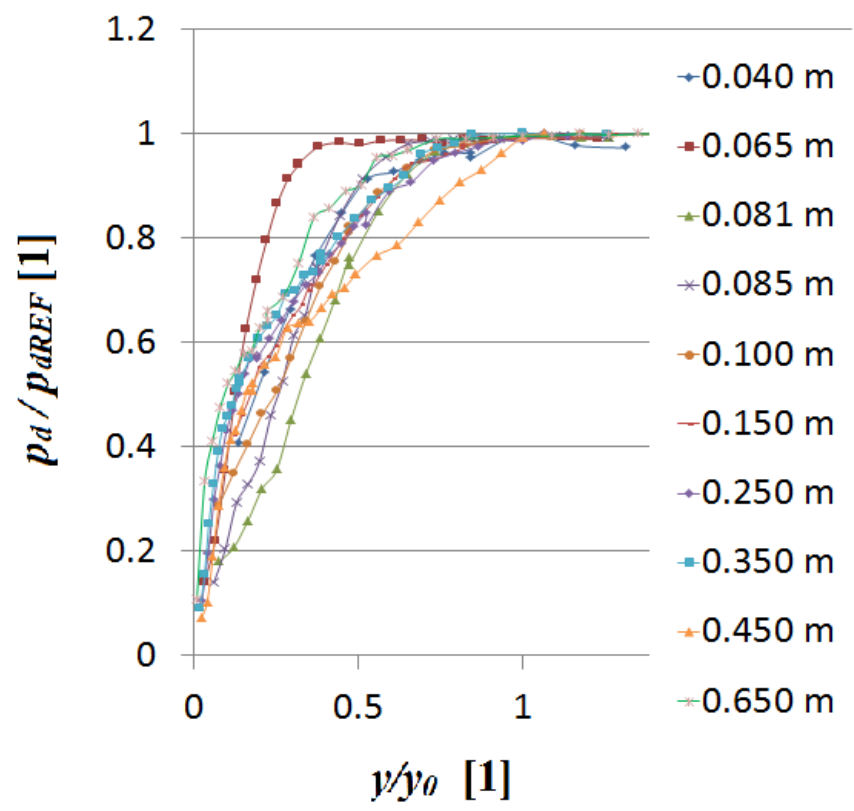

Figure 3: Shape of the $p / p_{\mathrm{d} R E F}$ profiles development along the boundary layer, example - case P32.

\subsection{Transition process}

Canonically, process of the boundary layer transition into turbulence is described as follows. Boundary layer starts from leading edge of the flat plate in stream wise direction. The process of laminar to turbulent flow transition starts in a specific distance from leading edge. It relates to the generation and propagation of turbulent spots, growth of the spots and mutual interactions, finally to full turbulent flow occurs.

This process can be described using intermittency coefficient (or "intermittency factor") $\gamma$. This coefficient is defined as time ratio of duration of the turbulent flow in specific point of boundary layer flow field - the flow is intermittent in time in the transitional zone. The transition onset is usually defined as a point $\left(x_{0}\right)$, where $\gamma\left(x_{0}\right)=0,01$ and the transition termination is defined as the point $\left(x_{0,99}\right)$ where $\gamma\left(x_{0,99}\right)$ $=0,99$, see Figure 4 . 


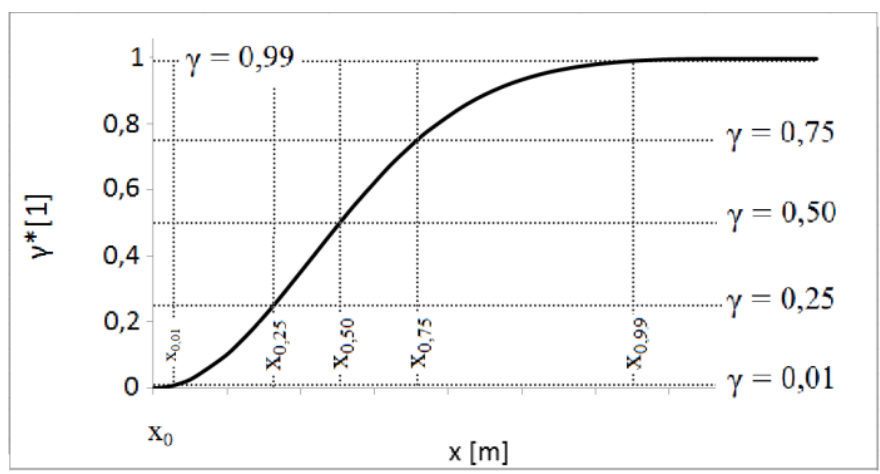

Figure 4: Canonically development of the boundary layer transition inside transitional zone, [8, 9].

Development of the intermittency coefficient $\gamma$ can be approximately derived from shape factor development based on Emmons hypothesis, [8]. The limitation is the linearity of this method. The principle lies in the observing of some integral boundary layer parameter $X$ development along the flow, such as boundary layer shape factor, skin friction coefficient, etc.

$$
X=(1-\gamma) X_{\mathrm{L}}+\gamma X_{\mathrm{T}}
$$

For some basic cases, such as flat plate boundary layer, value $X_{\mathrm{L}}$ for fully laminar boundary layer and value $X_{\mathrm{T}}$ for fully turbulent boundary layer are known, [8,9].

The transitional process can be observed as the development of the shape factor $H_{12}$ value along the flow. Onset of the transition process corresponds to the starting point of the shape factor $H_{12}$ decrease (ideally from $H_{12} \approx 2.6$ for fully laminar boundary layer). The finish of the transition process (fully turbulent flow) corresponds to the point where the shape factor $H_{12}$ stops to decrease (ideally $H_{12} \approx 1,4$ or 1,5). See example in Figure 6. The distributions of the shape factor $H_{12}$ can be used to statistically describe the transition process in the boundary layer, $[8,9]$.

The shape factor $H_{12}$ can be calculated as

$$
H_{12}=\frac{\delta_{1}}{\delta_{2}},
$$

where $\delta_{1}$ is the displacement thickness of boundary layer

$$
\delta_{1}(x)=\int_{0}^{\infty}\left(1-\frac{\overline{U(x)}}{\overline{U_{\infty}(x)}}\right) d y
$$

and $\delta_{2}$ is a the momentum thickness of boundary layer.

$$
\delta_{2}(x)=\int_{0}^{\infty} \frac{\bar{U}(x)}{\overline{U_{\infty}(x)}}\left(1-\frac{\bar{U}(x)}{\overline{U_{\infty}(x)}}\right) d y
$$

\section{Results of the experiment}

Number of preliminary measurements was carried out. Some basic characteristics of the boundary layer development (thickness, displacement thickness and momentum thickness, shape factor) along the flat plate are shown in Figures 5 - 7. Different roughness of strips in Figures 5-7 is denoted as P100 (100 particles $\left./ \mathrm{cm}^{2}\right)$ and $\mathrm{P} 32\left(32\right.$ particles $\left./ \mathrm{cm}^{2}\right)$. 


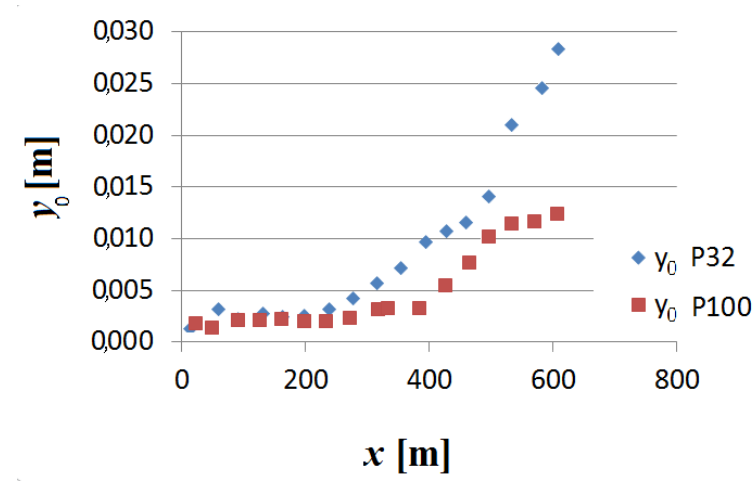

Figure: 5 Thickness of the boundary layer $y_{0}$ (defined as $u / U=0.99$ ) along the flat plate, roughness strip P32 vs. P100.

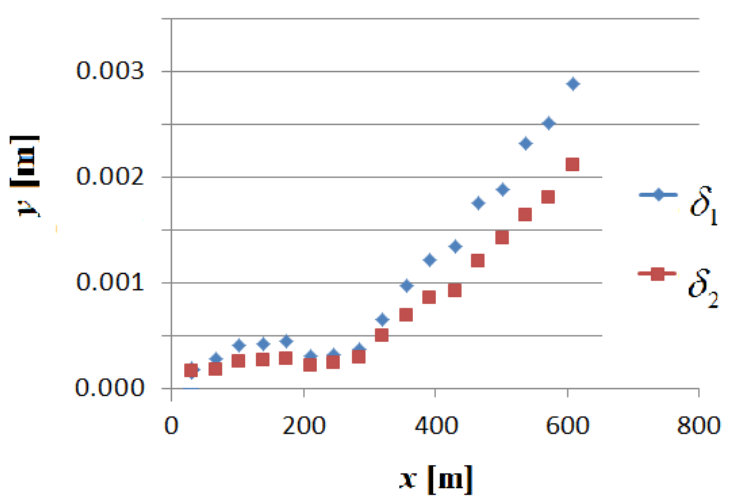

Figure 6: Displacement thickness $\delta_{1}$ and momentum thickness $\delta_{2}$ of the boundary layer along the flat plate, roughness strip P32 (Notice: Case P100 was not yet successfully analysed).

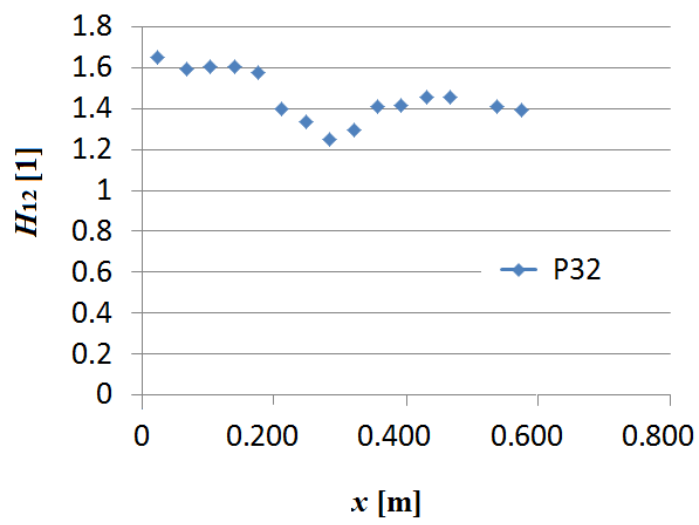

Figure 7: Shape factor $H_{12}$ in different points along the boundary layer, roughness strip P32. (Notice: Case P100 was not yet successfully analysed).

For example, see Figure 7 - development of the shape factor $H_{12}$. The evaluated behaviour of the boundary layer does not correspond to the canonical case of the transition of the boundary layer into turbulence. The oncoming flow is influenced by the rounded leading edge at the beginning of the plate, where the first point of the graph was placed. The second point was measured at half the depth of the roughness strip. The third point was measured on the smooth surface of the flat plate just behind the roughness strip. All other points in the graph were placed on the smooth flat plate (behind roughness 
strip). It can be observed that at the beginning of the monitored area the flow is not laminar. Rather it seems to be turbulent since the shape factor value does not reach 2.6. This is followed by decrease of the shape factor to 1.25 , which in turn denotes the turbulent shape of the mean velocity profile - the flow in boundary layer seems to be more turbulent. Further downstream, the value of the shape factor increases again. This can be explained by a attenuation of the turbulence. The effect of the rough strip is probably lost here.

\section{Conclusions}

Roughness strip is important and common task in fluid dynamics. Roughness strip (or some local noise on the surface) accelerates the transition process of boundary layer. Transition starts closer to the leading edge of the flat plate and the length of the transitional zone is shorter. Presented results are preliminary and will be refined. The time of one measurement of each point seems to be too short and will be changed, according to the proven stability of the observed results. Area close to the roughness strip will be examined in more detail. Some other data corrections will be applied (probe blockage effect close to the surface). Research for more cases and different conditions will follow.

\section{Acknowledgement}

This work was supported by the internal project 90131 of IT CAS 90131.

\section{References}

[1] J. Př́ihoda, P. Straka: Modification of the algebraic transition model for wall roughness effect including a rough strip. Proceedings Topical Problems of Fluid Mechanics 2020. (2020).

[2] M. Yang, Z. Xiao: Distributed roughness induced transition on wind-turbine airfoils simulated by four-equation k- $\omega-\gamma$-Ar transition model. Renewable Energy, vol. 135, no. 5:( 2019) pp. 11661177

[3] M. F. Kerho, M. B. Bragg: Airfoil boundary-layer development and transition with large leadingedge roughness. AIAA, vol. 35, no. 1: (1997) pp. $75-84$.

[4] R. S. Ehrmann: Effect of surface roughness on wind turbine performance. Disertation thesis. (1997).

[5] David C. Maniaci et al: Experimental measurement and CFD model development of thick wind turbine airfoils with leading edge erosion. Journal of Physics: Conference Series, vol. 753, no. 2: (2016).

[6] C. M. Langel et al: Analysis of the impact of leading edge surface degradation on wind turbine performance. 33rd Wind Energy Symposium: (2015).

[7] C. Tropea, A. Yarin, J. F. Foss: Springer handbook of experimental fluid mechanics. (2007) pp 230.

[8] H. W. Emmons: The laminar turbulent transition in boundary layer, J. Aero. Sci., 18: (1951) pp.490-498.

[9] R. Narasimha: On the distribution of intermittency in the transition zone of a boundary layer. $J$. Aero. Sci., 24: (1957) pp. 711-712. 